\title{
ESP 8266 Node MCU Based Weather Monitoring System
}

\author{
Sarmad Nozad Mahmood, Sameer Alani, Forat Falih Hasan, Mohammed Sulaiman Mustafa \\ \{sarmadnmahmood@gmail.com, itsamhus@gmail.com, forat.db@gmail.com,moh.kirkuk@gmail.com\} \\ Department of Computer Technical Engineering, Alkitab University, Iraq
}

\begin{abstract}
This paper primarily blends two-study fields based on control systems with data acquisition methodology and builds a database system to produce the data according to the chosen attributes. The sensors for weather station were used to quantify and store data about temperature, humidity, and wind speed. The collected data can be represented as direct and indirect in two forms owing to the regular reading and preservation of the data as a specific database system respectively. The new technologies for the development of databases are considered the main challenge of this research. The entire system tracks and controls positions centrally and remotely depending on the regular climate change that happens, to keep the desired positions in optimal environmental situations. The proposed device uses an ESP8266 node MCU microcontroller to manage and monitor the condition of the location whenever and wherever remotely controlled NET PI network system is used. The ESP8266 serves as an AP access point, connected to the network through a Wi$\mathrm{Fi}$ (router) station. Furthermore, the webserver can automatically and manually switch the devices ON/OFF through the NETPI platform. Finally, the weather information can be realized in the same manner and can be stored in an excel sheet simultaneously to be visualized via the $\mathrm{R}$ programming language.
\end{abstract}

Keywords: ESP8266 Microcontroller, Weather Monitoring System, Modular Control System, Temperature and Humidity Sensor, Net Pi Network Platform.

\section{Introduction}

The Internet of Things (IoT) is a series of computer, mechanical and non-electronic systems, remote communication network setup, service, and regulation. The person in charge can monitor the central and external devices, switch on / off machines, transfer circuits between multiple devices, access devices and remote controls, etc [1]. A weather station is considered a scientific tool that enables the calculation of weather parameters dependent on environmental conditions either on the soil or sea to predict weather conditions and analyze climate data. Forecasting officially started in the nineteenth century[2]. The collected data enables the chosen position to be determined and validated. Two factors, temperature and humidity are valued mostly for the environment. Such factors fluctuate especially in places that have been influenced by the intensity of the sun's radiation and the perpendicular angle of the sun that radiates over a specific location depending on the tropical line's latitude. In reality, the environment is rendered as a jumbled and vital scheme because of the tight-grade results which could be dumped over the whole scheme in the event of a tiny change $[3,4]$. Hence, the issue causes problems for farreaching temperature forecasts, as estimation could only be feasible for a few days to come. Weather station experts thus remain incessantly day by day forecasting climate situations based 
on the scientific analysis of atmospheric conditions. It must be noted that human experience in the past has followed certain criteria and terminology such as the calculation of heat, the analysis of humidity intensity and its impact on weather, environmental situation and eventually, the state of the atmosphere which seldom has an effect on the standards suggested. The centuries of development and smart devices that focus on microcomputers and microcontrollers have simplified the words considerably into efficient influences, appropriately coping with the change in environmental conditions. Adaptation of client inputs though is considered the greatest aspect concerning the arrangements for the expected output-based database model. The study describes three weather criteria used to produce and collect data packaging and focused on the conceptual weather station. These three environmental factors are Temperature (T), Humidity $(\mathrm{H})$ and Wind speed $(\mathrm{W})$. The lighting structure and Heating, Ventilation, and Air conditioning (HVAC) system are used to control the power output of the light bulbs and the HVAC system manually and automatically via a NET PI web server site. Due to the position region based on data structure conditions, the estimation procedure for realizing wind speed has faced a few challenges. Lastly, the concept is considered ideal particularly for monitoring specific devices dependent on sensor readings through the NET PI network platform and the implementation was done at ALKITAB University, Iraq.

\section{Literature Review}

The IoT uses a wide variety of devices, protocols, technologies, networks, middleware, applications, systems, and data, all forming a heterogeneous network. This will increase the degree of interoperability and complexity. Because of this situation, several groups such as ITU, ETSI, OpenIoT are developing interoperability standards and IoT protocols, among others. However, the high fragmentation and development of vertical IoT systems have increased in a multi-standard context, where features, functions and devices are combined [5]. In [6] an energy and position-based IoT system are controlled in networks, which are also based on a smartphone and cloud computing platform. This provides energy efficiency in buildings and organizations, as a complete system on a large scale. The problem with this proposal is that it is difficult and costly to implement, so it is not recommended to be applied in ordinary smart houses. A conceptual IoT device called an AAL-IoTSys, which includes a Smart Gateway as a key component is proposed in [5]. This enables many heterogeneous devices to be interoperable across different networks, protocols and technologies eg. WiFi, ZigBee, Bluetooth, IEEE 802.15.4 and 6LowPAN. Many research papers and studies in this area do not emphasize microcontroller sensor outputs for data storage and data acquisition. In this article, the resulting data collected from the device can be concurrently processed and charted synchronously with a weather station monitoring system [3]. That is, the details can be shown and seen, directly and indirectly, in two approaches. The word 'direct' implies that the weather can be viewed directly via the NET PI network platform; whereas indirect methodology ensures that weather patterns are recorded and stored in a computer as long as the sensors calculate climatic conditions [3, 7]. The main difficulty of this research is to demonstrate and validate that microcontrollers can be connected to a data acquisition network with their sensors to build a database system based on the characteristics of the weather. The current idea helps the microcontroller sensors to predict possibilities centred on the perceived data rather than strictly tracking the device. Economically, a single sensor known as DHT sensor is used by the proposed system to provide temperature and humidity readings that were used to create the heretical framework of the climate database [2]. The articles discussing the concept of green solar systems and hybrid power plants addressed many causes that have a significant impact on appreciations of the weather conditions, 
such as the colour of sun rays due to environmental reactions and vibrations that alter the temperature situation and thus adjust the humidity situation proportionally $[8,9,10]$. In turn, wind speed is also another aspect that has an enormous influence on climatic conditions such as airspeed, air intensity, air direction and air coldness. The circumstances and variables suggested have a significant effect on the human being's daily weather outlook. This paper provides a simple way of remotely tracking/storing the data, that is, the consumer should install the application at a specific location and start recording and storing the data.

\section{ESP8266 Node MCU}

A Node MCU is a modern microcontroller piece that had been created by Arduino Enterprises. This piece works as Arduino with requirements irrespective of AVR processors $[11,12]$ that excite Arduino IDE C++ compilers to compile the whole packet. Thanks to the features implemented by the ESP team, the package is considered to be a complete kit intended to reduce the specific sectors that are required to be linked to various roles of the perform. The term ' Core ' has been given to the software group which is needed to debug the Arduino C++ headers using MCU language. The innovation of the ESP8266 module contributes to the creation of robust and complete systems as opposed to the design methodology that developed the Arduino core under the hegemony of the ESP8266 Wi-Fi based on GitHub ESP8266 core website. This module is a platform for machine learning, incorporating between ESP8266 and NodeMCU. The unit shown in Figure 1 operates under the control of networks 802.11n and 802.11b. This means it can be used as an access point AP and Wi-Fi system or both together [13] simultaneously.

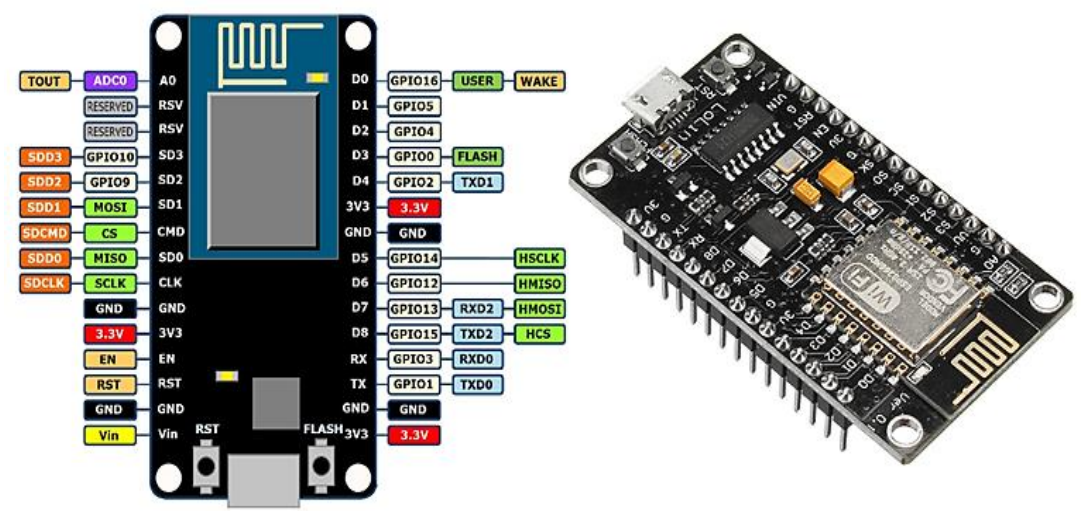

Fig.1. ESP8266 NodeMCU Microcontroller

\section{Temperature - Humidity Sensor (DHT11)}

The Temperature-Humidity sensor known as DHT11 detects and controls temperature and humidity degrees in a single distinctive configuration. The DHT11 sensor describes the temperature $(\mathrm{T})$ and humidity $(\mathrm{H})$ forms which are handled in a complex manner using optical signal output adjustment. The sensor promises outstanding flexibility and excellent long-term reliability, owing to the private digital signal processing in the sensing devices. This unit involves a resistive humidity circuit alongside with an NTC temperature pad connected to an 8bit high-performance microcontroller used to provide excellent efficiency, quick response, anti- 
interference functionality and cost benefit-effectiveness [12, 14]. The DHT11 sensor tests both (T) and $(\mathrm{H})$ in this project and transmits the readings via the ESP8266 board to the Net Pie network platform. The unit consists of three pins identified by Vcc, data, and Gnd. it has to be mentioned that the system exhibits excellent innovations especially when connected to any digital pin in the microcontroller. As shown in Figure 2 [4, 15], the VCC pin must be supplied by $5 \mathrm{~V}$ of ESP8266 MCU, the data and the Gnd are connected to the digital pin GPIO2 (D4) and the Gnd pin of the Node MCU respectively.

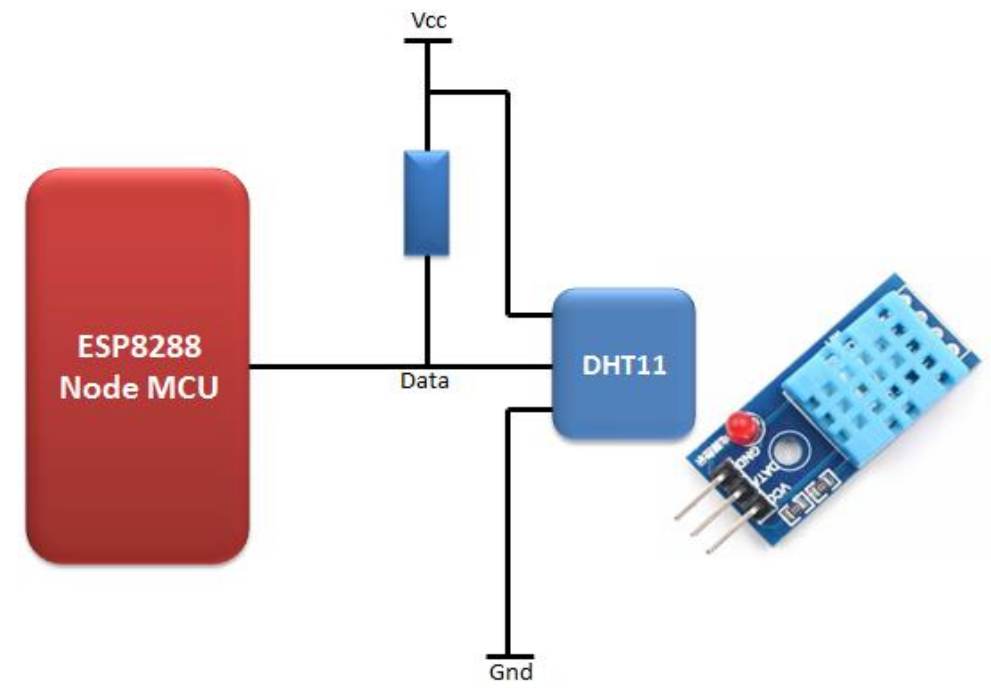

Fig.2. Temperature and Humidity DHT11 Sensor

\section{Wind Speed Meter (Anemometer)}

An anemometer is a tool used to determine wind speed and has been deemed an important instrument for the weather station [4]. The anemometer is intended to be mounted outdoors to transmit the signal easily to the microcontroller. Nonetheless, most research papers and programs are not incorporating such a responsive feature as the wind speed sensor suggested. This sensor is a supreme item that incorporates effectively with weather monitoring components. In this study, wind speed is assessed, processed and viewed directly on the proposed network platform at a local specific location. In contrast, the Arduino code is recommended to indicate the highest wind speed determined so that if the wind blows hard in a given moment, the maximum wind intensity every period is recorded and shown in the section allocated to calculate the wind's peak level. The average intensity of the wind varies every once in a while depending on the actual wind speed expected to be recorded in the current database system and shown on the NET PI platform as long as there is no greater value than the last read. The operating theory of this fantastic wind speed sensor is strongly dependent on the precise construction of the anemometer. The device's internal construction is made of a DC motor that operates synchronously with the movement of the spinning cups as shown in the right side of 
Figure 3. Owing to the pole arrangement between the motor and the hub holding the cups, the spinning of the revolving cups allows the DC motor to rotate synchronously. Note that the rotation and energy in the DC motor system are inversely proportional, indicating that the motor will produce mechanical motion when power is given and can act as a voltage generator when the rotor is activated by mechanical torque. Accordingly, the anemometer is connected to the analogue pin of the microcontroller.
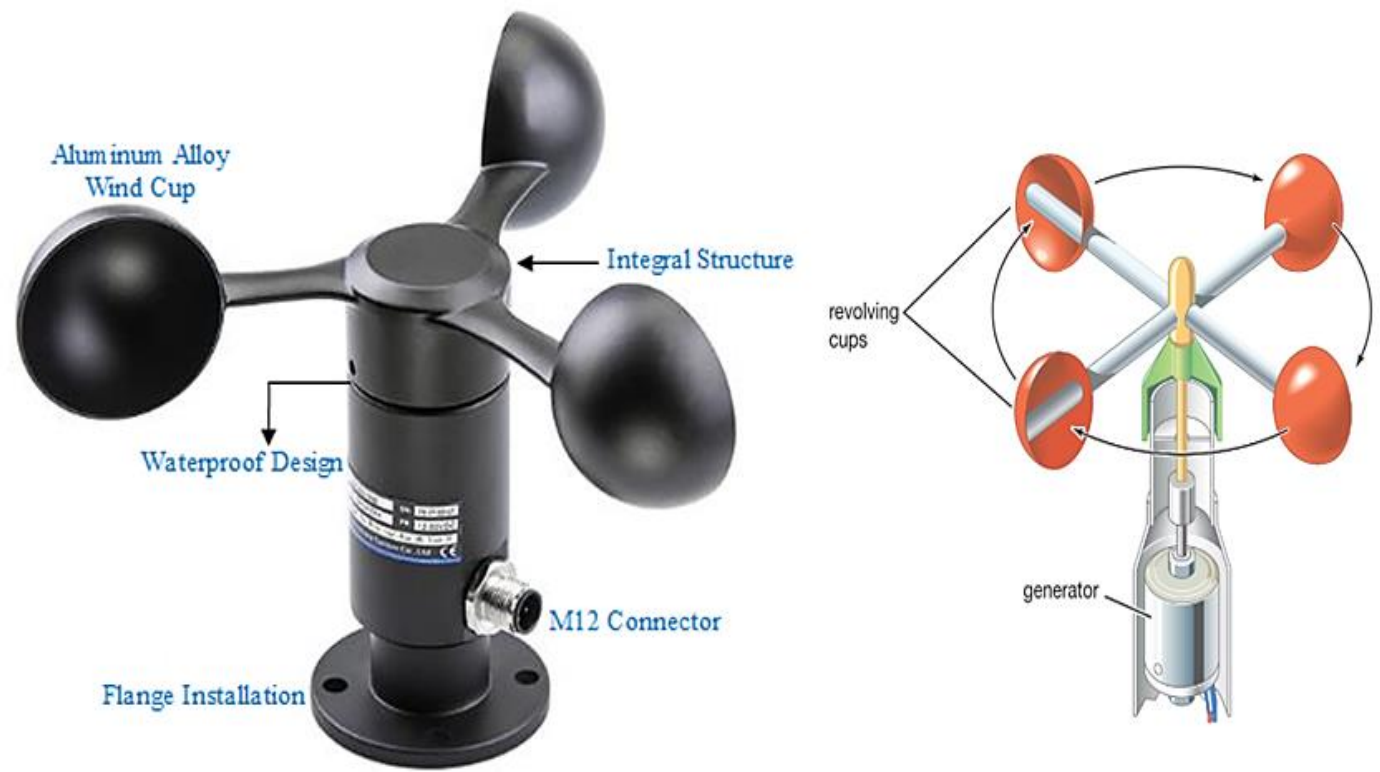

Fig.3. Wind Speed Sensor (Anemometer)

The anemometer's output terminals consist of three wires defined by Vcc, GND, and Signal cable. It is worth mentioning that the proposed anemometer needs external isolated power within a range of $9-12$ volts $[4,15]$. The terminal ( $\mathrm{Vcc}$ ) is connected to the battery's positive terminal, the terminal (Gnd) is attached to the battery's negative side as a common point with NodeMCU's (Gnd) and lastly, the signal link is attached to the analogue pin (ADC0) of the NodeMCU board as the diagram illustrated in Figure 4. 


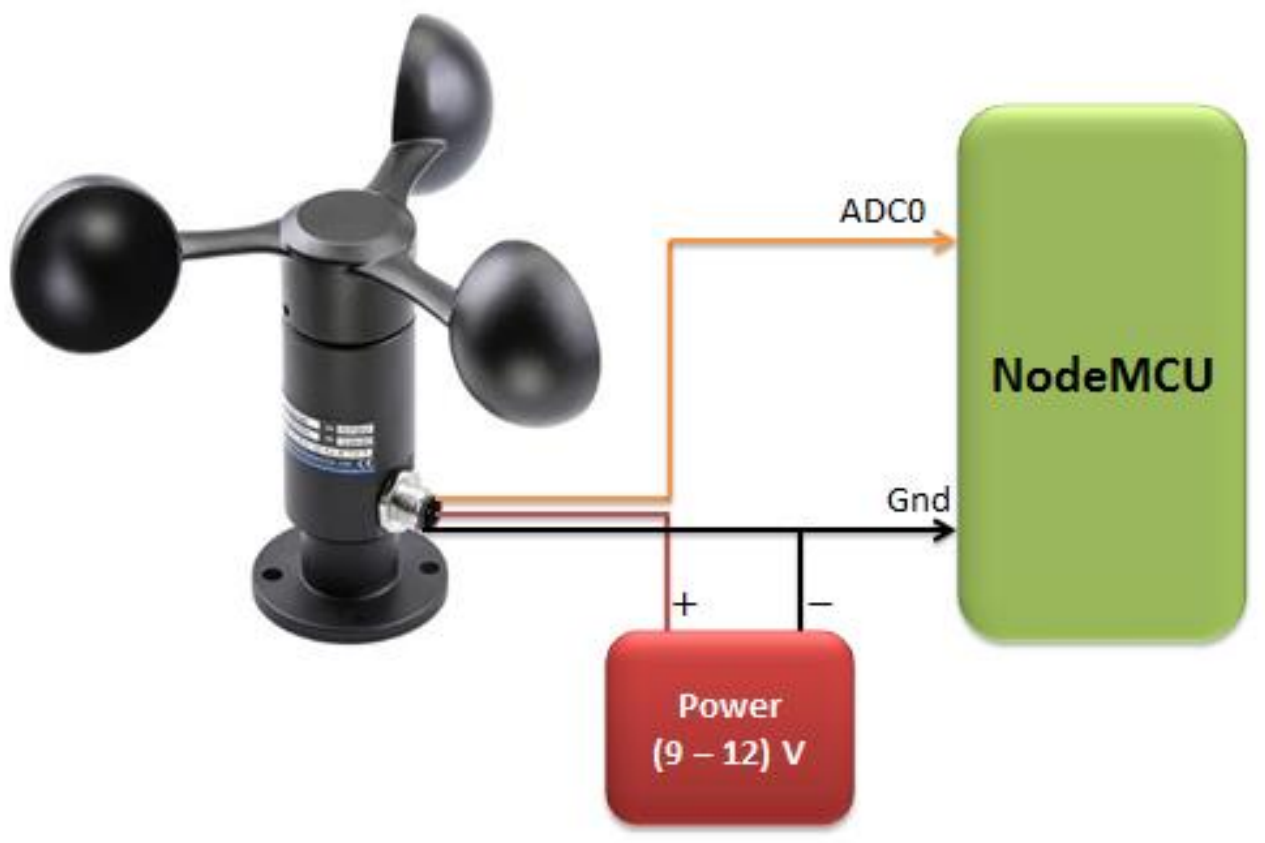

Fig.4. Anemometer Connection Technique

The voltage produced by the rotation of the cups is passed to the NodeMCU's analogue terminal, and therefore the wind speed is determined based on definite formulas defined in the code.

\section{R Language-Based Data Visualization}

The R-language based on data science complies with high statistical analysis and supports SQL functions according to R- Language packages. Noteworthy that Open Source Package Systems R-Language defines the most common language contributing to effective data analysis. This language allows the developer to create a mix as an interface between several programming languages to achieve optimal efficiency. Also, the language presented provides a significant potential to visualize data in different aspects $[16,17]$. The major reason for using this language can be fragmented into several reasons identified by smart management, reliable information and simple data realization $[4,18]$.

\section{Relay Based Devices Control}

The relay component employed controls in-house actuators and light sources [7sar\&for]. The relay is essentially the component element that is activated by $5 \mathrm{~V}$ input voltage, which 
implies that $0 \mathrm{~V}$ matches 0 and $5 \mathrm{~V}$ matches 1 [4]. The key contest that takes place based on the relay used in this conceptual smart house system is an Ac power transfer. The Node MCU adjusts the distinct switching states digitally, depending on the electrical signals sent from the MCU to the relay input to control the devices and the lights in the household environment. The relay is designed to connect/disconnect the NC (Normally Closed) port to C (Common) port, depending on the pulses transmitted from the digital pin connected to the MCU. It is proposed to activate the actuator defined by the HVAC such that the cooling system switched ON whenever (T) gets greater than a particular range and the heating system switched ON when (T) is less than another range based on the code of MCU. Despite this, the actuators stay inactive as long as (T) lies between the scopes that the model prefers. Eventually, in Figure 5, the connection scheme reveals the elements provided under relay control along with an AC light source. Simultaneously, the entire system can be controlled manually when the user desires to monitor the environment remotely or even to control the devices anytime and anywhere.

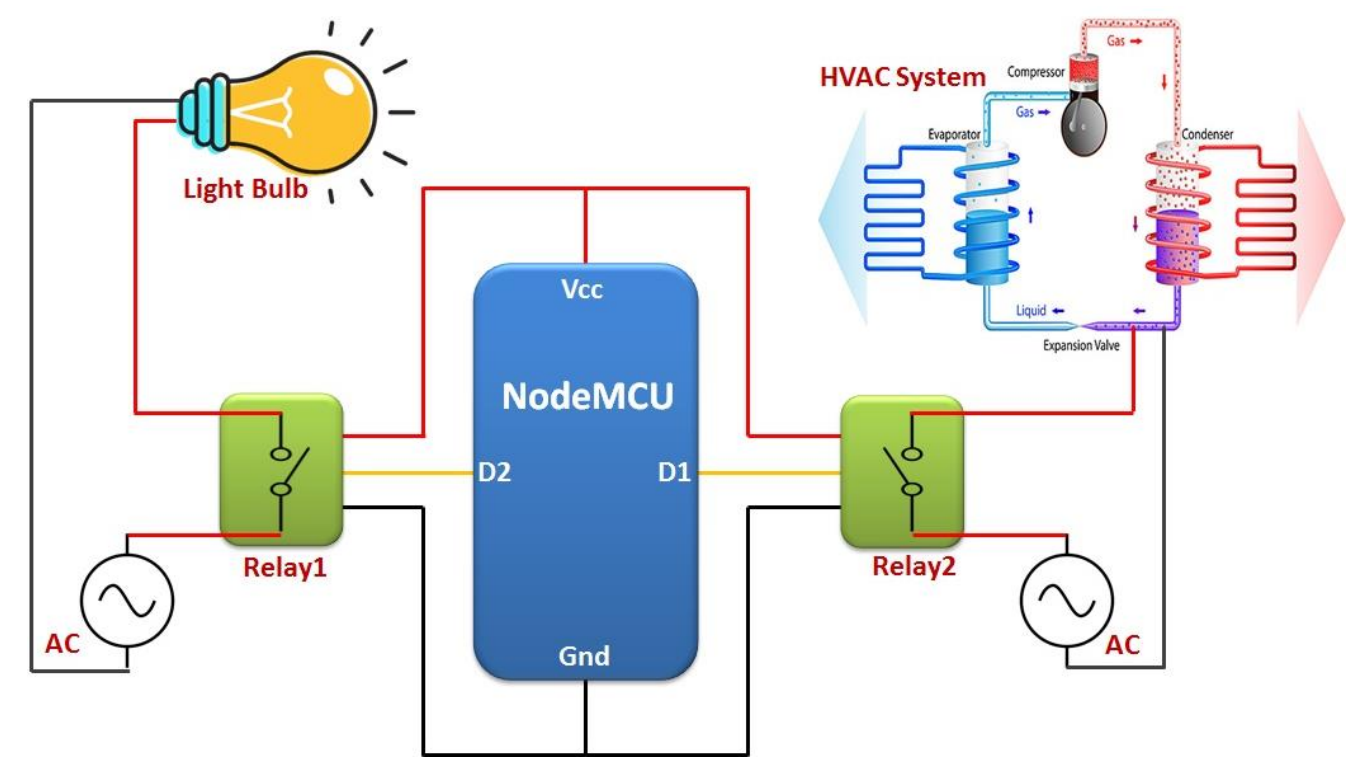

Fig.5. HVAC and Lighting System Connection

\section{The Methodology}

The system's primary dictator denoted by node MCU manages each part separately. The temperature $\mathrm{T}$ and the humidity $\mathrm{H}$ are measured by a DHT11 sensor through the digital pin D4 of node MCU and are shown periodically online via the NET PI network platform (https://netpie.io/) alongside the speed of the wind across the analogue pin ADC0 in the MCU. Moreover, the system contains two relay (contactor) modules which can be activated when 
provided by 5V PWM from the microcontroller. The first relay (Relay1) is responsible for controlling the flow of the AC voltage to the HVAC system through pin D1 of the MCU. The second relay (Relay2) switches the light bulbs of the building ON/OFF through pin D2 as shown in the connection diagram in Figure 6. The situation is presented to meet system designerprepared control requirements. Configuration requirements give the sensors preference, specifically the sensors responsible for the characteristics of weather stations. The triggering of the actuators depends on the reading of the DHT sensor so that the cooling system (HVAC) is switched $\mathrm{ON}$ when $\mathrm{T}$ gets higher than $28^{\circ} \mathrm{C}$ and OFF otherwise, regardless to the manual control processing. Similarly, when T gets less than $18^{\circ} \mathrm{C}$, the heating system (HVAC) is switched ON. Consequently, the HVAC in the range of $18-28^{\circ} \mathrm{C}$ is completely idle. Finally, the paper presents a great challenge by creating a common accumulation system to link node MCU with data acquisition software and store the main outputs of each sensor separately in a private excel sheet via the IDE code given by the ESP8266 based node MCU and NET PI network platform.

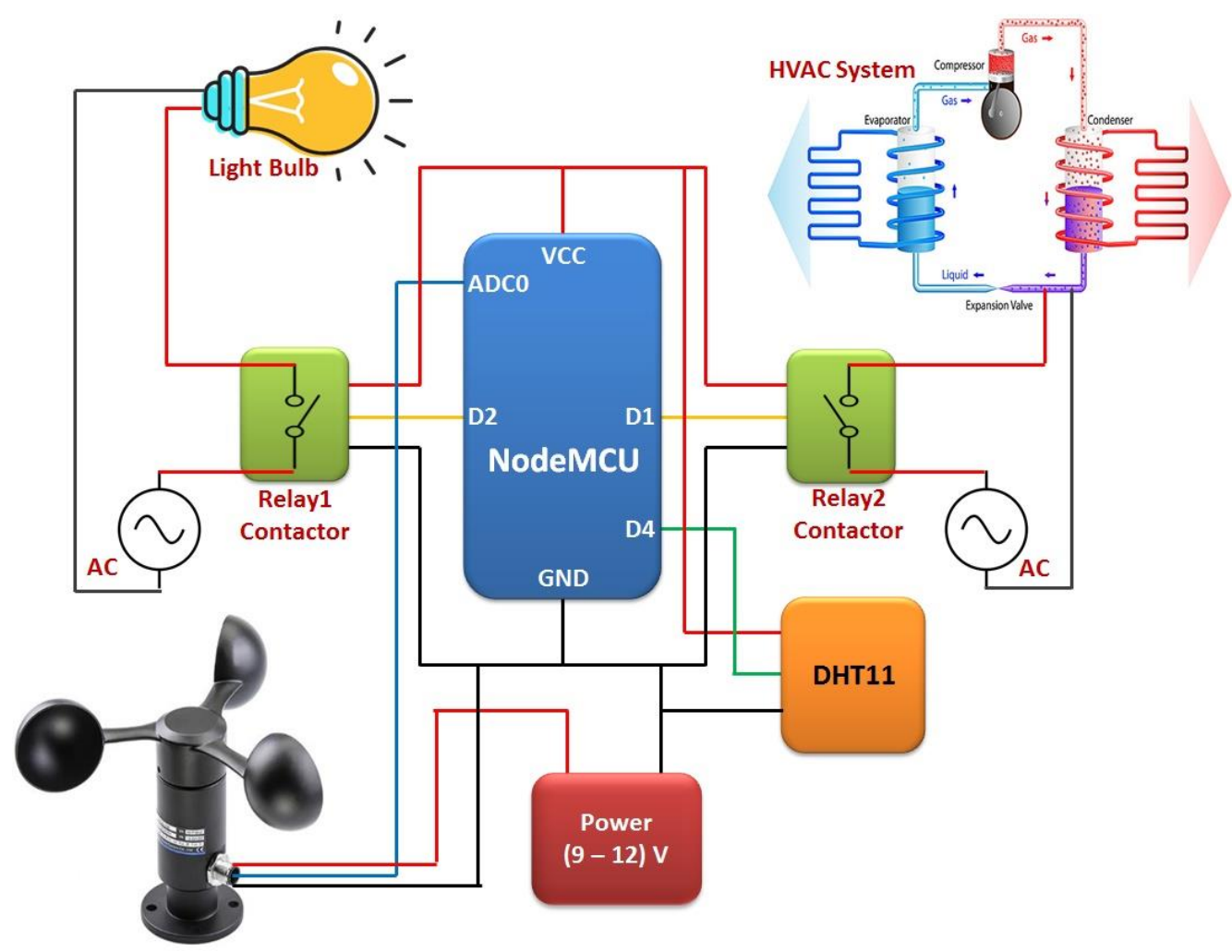

Fig.6. The Overall System Connection Diagram

\section{System Coding}


The system starts calling the libraries required to configure DHT11 alongside with the other components. Firstly, the entire sensor components are activated once they are provided by the input voltage from node MCU. Then, the initial condition and the maximum value of the wind speed are computed depending on the following set of equations [4]:

$$
\begin{gathered}
\text { Map }(\text { Min Volatge }- \text { Min Speed })=\operatorname{Map}(0.01-0) \\
\text { Map }(\text { Max Volatge }- \text { Max Speed })=\operatorname{Map}(1.30-32)
\end{gathered}
$$

The whole computation needs to be adapted with the analogue terminal of the microcontroller to calculate the difference between the analogue and digital read range defined by $0-1023$ and $0-5$ respectively. The adaptation factor can be realized by the division of the digital to the analogue range which gives approximately 0.005 . The remaining part of the computation will be given as follows:

$$
\text { Sensor Voltage }=(A D C 0) \text { Value } * 0.005
$$

Where $(A D C 0)$ is the analogue port of the MCU. The system software is conveniently explained through the flow chart diagram shown in Figure 7 [19, 20]. Also, the flow chart simulates the loop portion of the proposed programming language of the method, which begins by reading the sensors to realize the values of their corresponding variables. Instantly, the DHT sensor and the anemometer will start measuring the temperature, humidity, and speed of the wind respectively. Through the node MCU microcontroller board, the received data are stored in the excel sheet through a specific windows program. After this stage, a user starts to regularly supervise the system based on the required criteria. Many decisions are taken into consideration in this method to run the system dynamically depending on the recognized data so that the HVAC operating system takes place accordingly. Furthermore, the lighting system in the building runs in the same manner and both modules satisfy the required specification of the control system. 


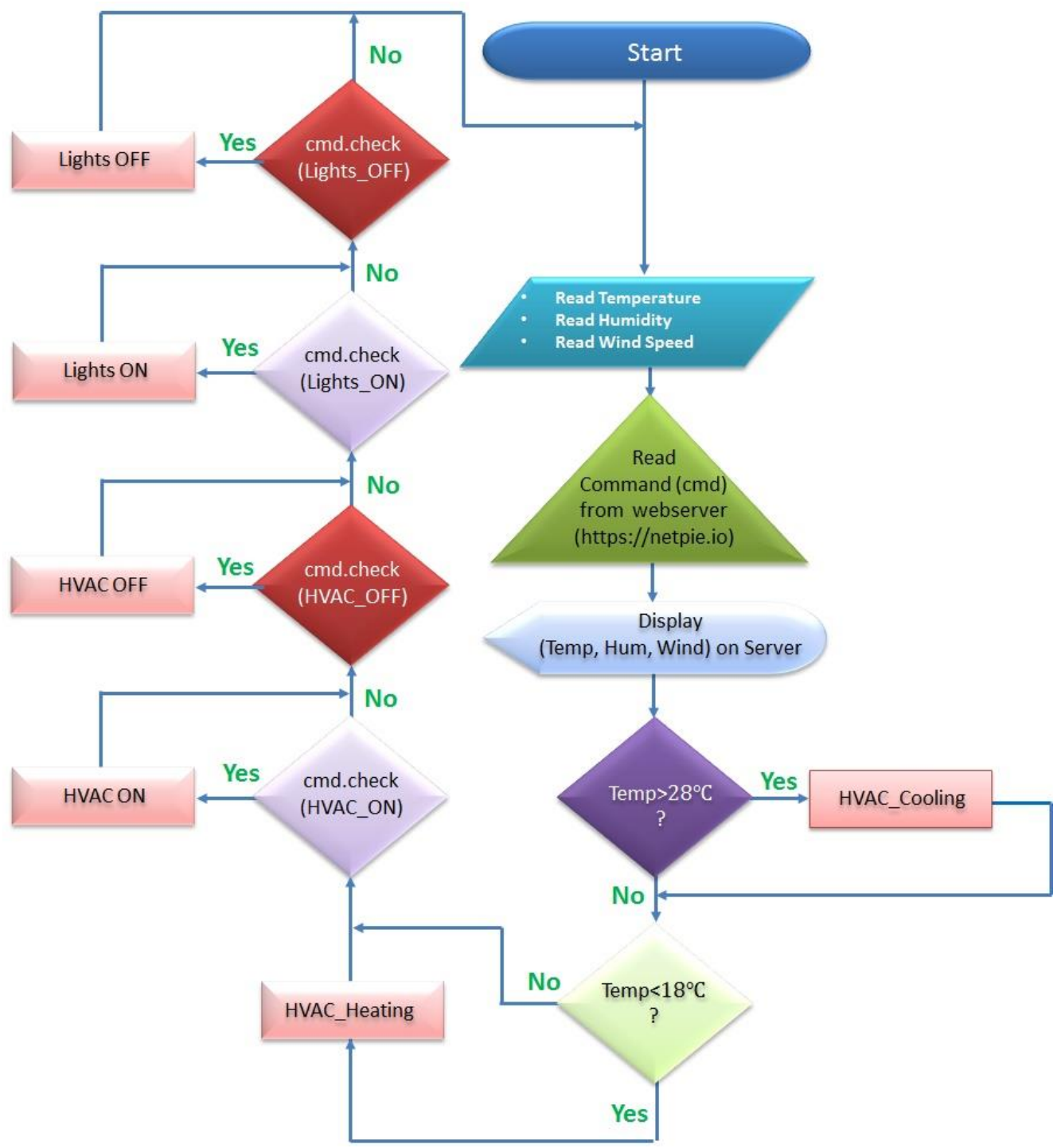

Fig.7. Programming Code as Flowchart

The term HVAC applies to the heating, ventilation and air conditioning systems or even the house lighting system, both of which are expected to be manually or remotely operated based on certain factors. The architecture of the control system is considered crucial especially when dealing with high AC voltages (220V). Nevertheless, this project's inspiration was effectively achieved using the NET PI (https://netpie.io/) web server control system focused on the ESP8266 microcontroller, as shown in Figure 8. 


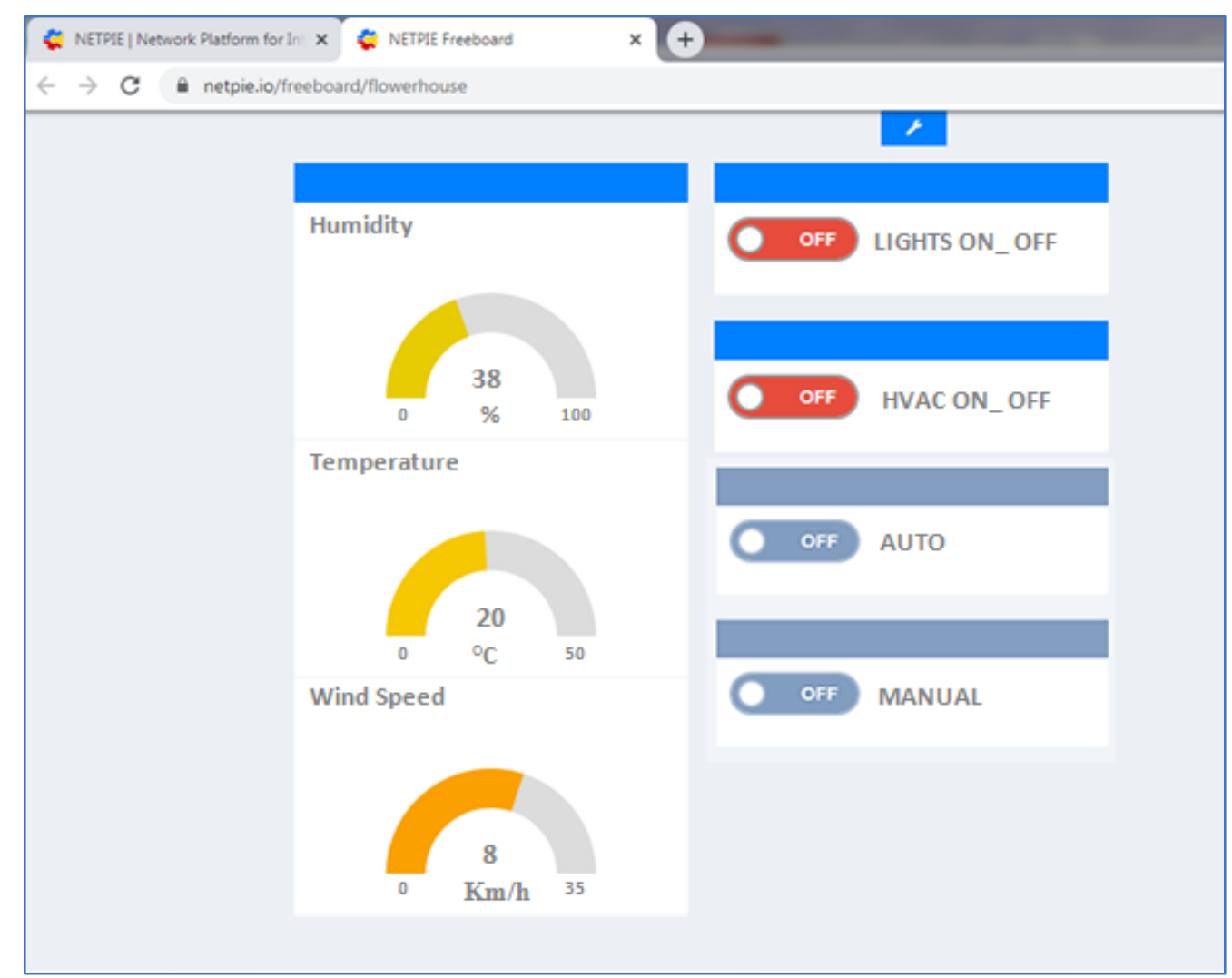

Fig.8. Real Results on Net PI Platform

It is noteworthy that the climate conditions were stored at Alkitab University on the $30^{\text {th }}$ January 2020 for 12 hours at the ALKITAB University which is located at 35.761945latitude and 44.158311longitude. As confirmation, it is intended to demonstrate the realized data using R- a programming language in the form of carves reviled for temperature ( $\mathrm{T}$ ) in Figure 9, for Humidity (H) in Figure 10, and Wind Speed (W) in Figure 11.

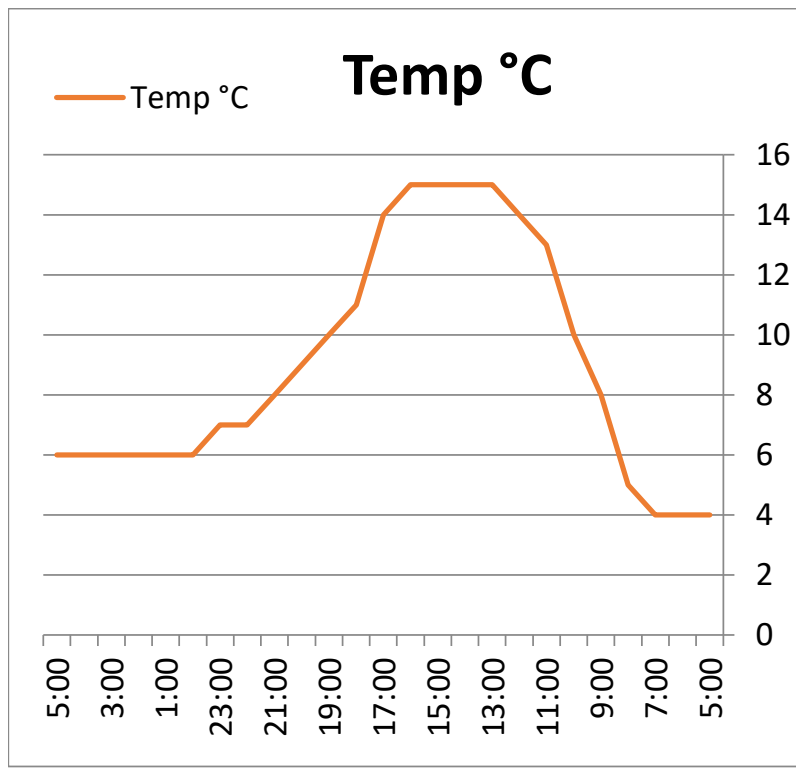

Fig.9. Temperature Data

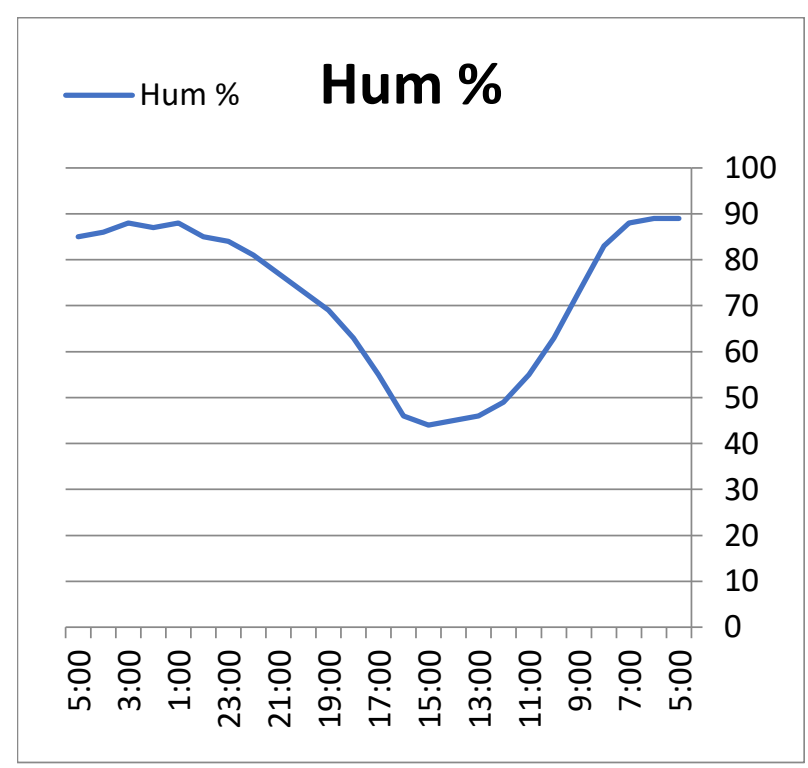

Fig.10. Humidity Data 


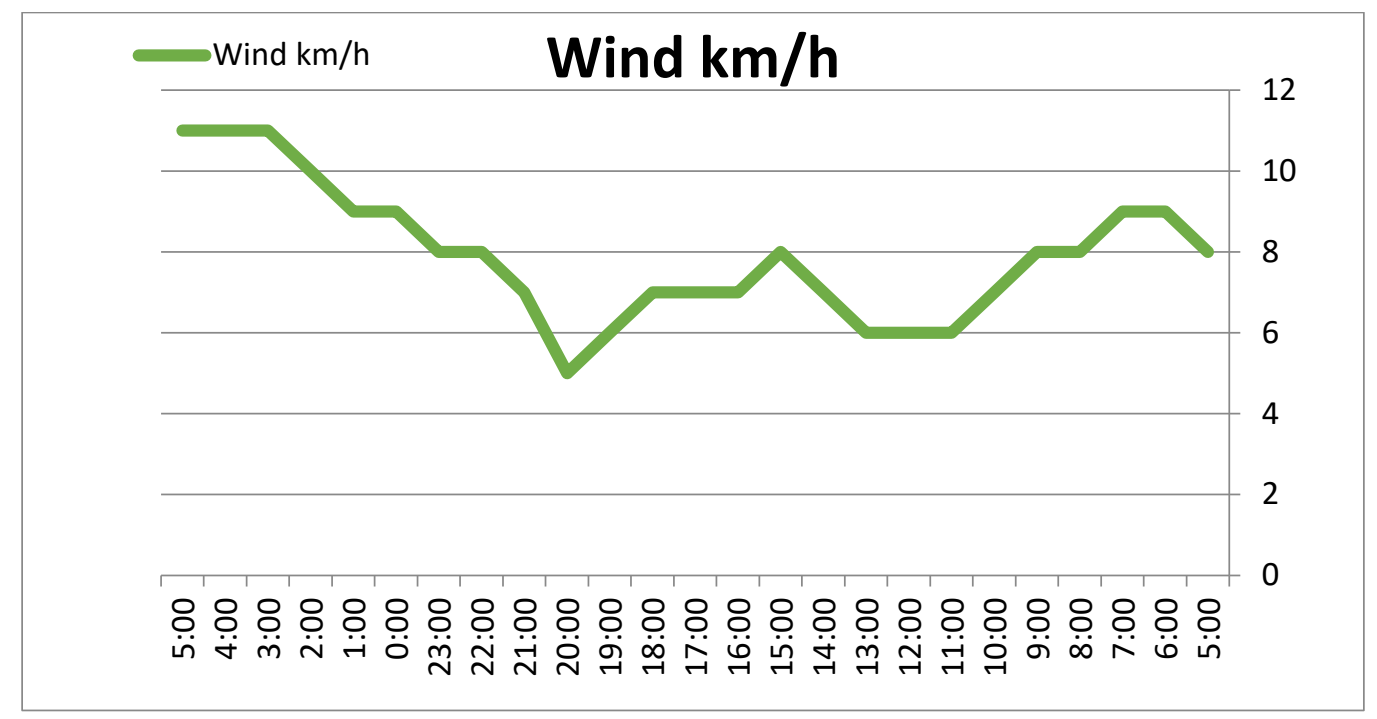

Fig.11. Wind Speed Data

Finally, the relationship between the overall possible weather components is unveiled in the form shown in Figure 12 to perceive the weather status unambiguously.

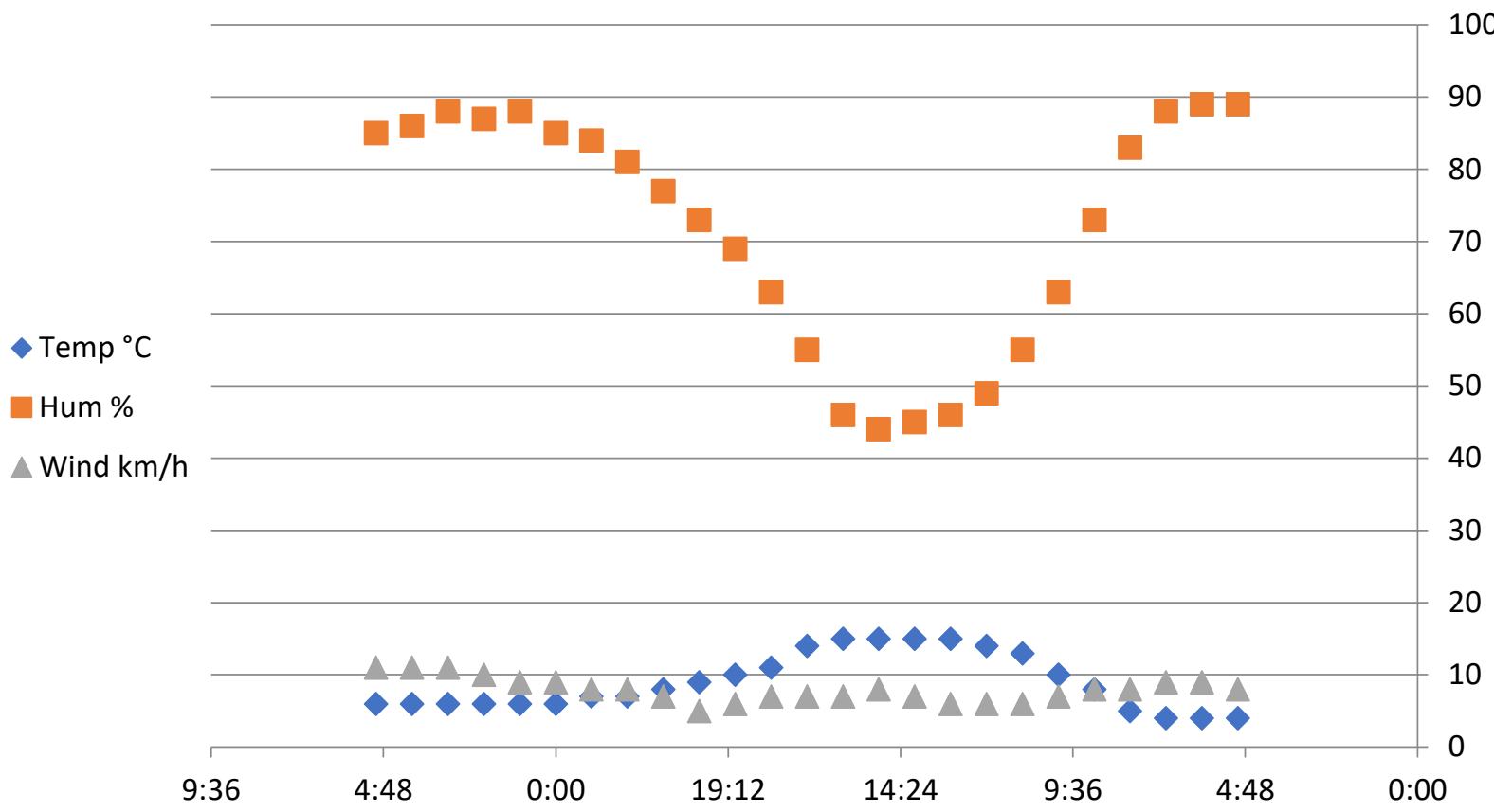

Fig.12. Overall Weather Data

\section{Conclusion}

This paper presents an innovative and dependable concept of a low-cost simple weather monitoring and controlling system. The system operates under IoT technology supervision which effectively optimizes remote areas. The creativity of this revolutionary weather station allows monitoring and controlling of the web server-based climate conditions using the ESP8266 node MCU microcontroller. The outputs of the measurements employed are meant to be shown via the NET PI web server as adjustable gauges. In terms of network connectivity, the devices can be turned $\mathrm{ON}$ or OFF at any moment and anywhere. The complete dependence on the webserver control system and the applicability of the local IP given by the ESP8266 means that the design's cost is inexpensive. The system contributes to being applicable in two fields. 
The first contribution is extremely useful to businesses and other organizations that are tasked with preparing and handling their operations based on weather situations; such as high-priority transport systems, airways, and forestry, etc. The second contribution is specifically designed to control locations regarding the changes in user interface status based on information generated by improvements in output due to weather disturbances; such as monitoring residences, stores, hospitals, universities, and smart vehicles.

\section{References}

[1] Dhanalaxmi, B., \& Naidu, G. A. (2017, February). A survey on design and analysis of robust IoT architecture. In 2017 International Conference on Innovative Mechanisms for Industry Applications (ICIMIA) (pp. 375-378). IEEE.

[2] Krishnamurthi, K., Thapa, S., Kothari, L., \& Prakash, A. (2015). Arduino based weather monitoring system. International Journal of Engineering Research and General Science, 3(2), 452-458.

[3] Sabharwal, N., Kumar, R., Thakur, A., \& Sharma, J. (2014). A Low-Cost Zigbee Basedautomatic Wireless Weather Station With Gui And Web Hosting Facility. International Journal of Electrical and Electronics Engineering, 1.

[4] Mahmood, S. N., \& Hasan, F. F. (2017). Design of weather monitoring system using Arduino based database implementation. Journal of Multidisciplinary Engineering Science and Technology (JMEST), 4(4), 7109.

[5] Yacchirema, D. C., Palau, C. E., \& Esteve, M. (2017, January). Enable IoT interoperability in ambient assisted living: Active and healthy ageing scenarios. In 2017 14th IEEE Annual Consumer Communications \& Networking Conference (CCNC) (pp. 53-58). IEEE.

[6] Pan, J., Jain, R., Paul, S., Vu, T., Saifullah, A., \& Sha, M. (2015). An internet of things framework for smart energy in buildings: designs, prototype, and experiments. IEEE Internet of Things Journal, 2(6), 527-537.

[7] Gahlot, N., Gundkal, V., Kothimbire, S., \& Thite, A. (2015). Zigbee based weather monitoring system. The International Journal of Engineering and Science, 4, 61-66.

[8] Mahmood, S. N., Abdulabbas, A., Easa, H., \& Algburi, S. S. (2017, April). The battery characteristics impact on solar systems: Performance and cost. In 2017 International Conference on Current Research in Computer Science and Information Technology (ICCIT) (pp. 151-156). IEEE.

[9] Mahmood, S. N., Hussain, A. S. T., Easa, H. K., \& Algburi, S. S. (2017, August). Wind turbine power evaluation based on performance and cost factors. In 2017 IEEE 3rd International Conference on Engineering Technologies and Social Sciences (ICETSS) (pp. 1-6). IEEE.

[10] Hussain, A. S. T., \& Mahmood, S. N. (2017, August). Hybrid power station act based on runoff river. In 2017 IEEE 3rd International Conference on Engineering Technologies and Social Sciences (ICETSS) (pp. 1-7). IEEE.

[11] Vijay, Rampeesa, Thotakura Sainag, and A. Vamsee Krishna. "Smart Home Wireless Automation Technology using Arduino based on IOT." IJECT Journal 8, no. 4 (2017).

[12] Aziz, D. A. Design of Smart House System based on Webserver Architecture Control. International Journal of Computer Applications, 975, 8887.

[13] Saputra, L. K. P., \& Lukito, Y. (2017, November). Implementation of an air conditioning control system using the REST protocol based on NodeMCU ESP8266. In 2017 International 
Conference on Smart Cities, Automation \& Intelligent Computing Systems (ICON-SONICS) (pp. 126-130). IEEE.

[14] Laksono, P. W., Jauhari, W. A., Iftadi, I., Ayu, K. C., Pandu, B. I., Jamaluddin, A., \& Haijunowibowo, D. (2015, November). A system based on a fuzzy logic approach to control humidity and temperature in fungus cultivation. In Proceedings of the Joint International Conference on Electric Vehicular Technology and Industrial, Mechanical, Electrical and Chemical Engineering (ICEVT \& IMECE) (pp. 344-347). IEEE.

[15] Mahmood, S. N. (2018). GSM Interaction Based Real-Time Climate Change Monitoring Technique. Kirkuk university journal for scientific studies, 13(2), 1-17.

[16] Zhang, Y., Ordonez, C., \& Cabrera, W. (2016, May). Big data analytics integrating a parallel columnar DBMS and the R language. In 2016 16th IEEE/ACM International Symposium on Cluster, Cloud and Grid Computing (CCGrid) (pp. 627-630). IEEE.

[17] Harikumar, S., Haripriya, H., \& Kaimal, M. R. (2013, December). Implementation of projected clustering based on SQL queries and UDFs in relational databases. In 2013 IEEE Recent Advances in Intelligent Computational Systems (RAICS) (pp. 7-12). IEEE.

[18] Mishra, B. K., Hazra, D., Tarannum, K., \& Kumar, M. (2016, November). Business intelligence using data mining techniques and business analytics. In 2016 International Conference System Modeling \& Advancement in Research Trends (SMART) (pp. 84-89). IEEE.

[19] Ishak, A. J., \& Mahmood, S. N. (2019). Eye in hand robot arm based automated object grasping system. Periodicals of Engineering and Natural Sciences, 7(2), 555-566.

[20] Mahmood, S. N., Ishak, A. J., \& Hussain, S. T. (2019). GSM based gas leak monitoring system. Periodicals of Engineering and Natural Sciences, 7(2), 670-678. 